\title{
PERCEPÇÃO DO PACIENTE ONCOLÓGICO E DE SEU ACOMPANHANTE SOBRE CUIDADOS PALIATIVOS ANTES E APÓS INTERVENÇÃO EDUCATIVA
}

\author{
PERCEPTION OF ONCOLOGICAL PATIENTS AND THEIR CAREGIVERS ON PALLIATIVE CARE BEFORE \\ AND AFTER EDUCATIONAL INTERVENTION
}

\begin{abstract}
Clarisse Kaori Fujishige ${ }^{1}$, Danielle Yumi Akaishi ${ }^{1}$, Isabela Pereira Blanco ${ }^{1}$, Mariana Carvalho Gouveia ${ }^{1}$, Marília Arrais Garcia ${ }^{1}$, Roberta Ferraz Salles Kesselring ${ }^{1}$, Cláudia Vaz de Melo Sette ${ }^{2}$, Auro del Giglio ${ }^{3}$, Daniel de Iracema Gomes Cubero ${ }^{4}$,
\end{abstract}

Como citar: Fujishige CK, Akaishi DY, Blanco IP, et al. Percepção do paciente oncológico e de seu acompanhante sobre cuidados paliativos antes e após intervenção educativa. Clin Onc Let. 2018;3(1-2):2-10. https://doi.org/10.4322/col.2018.009

\begin{abstract}
RESUMO
Objetivos: Avaliar o entendimento de pacientes oncológicos e seus acompanhantes acerca dos cuidados paliativos, elaborar cartilha educativa e testá-la na mesma população. Métodos: 0 estudo foi dividido em duas etapas: diagnóstica e de intervenção. Na primeira, aplicou-se um questionário a pacientes oncológicos e seus acompanhantes a fim de avaliar seu nível de conhecimento acerca dos Cuidados Paliativos. Com base nas respostas obtidas, uma cartilha educativa no formato de gibi foi elaborada. Na segunda etapa, as cartilhas foram entregues a novos indivíduos, aplicando-se a seguir o mesmo questionário utilizado na primeira etapa. Resultados: Foram incluídos 194 participantes na primeira etapa e 50 na segunda. Não houve diferenças significativas nas características dos sujeitos de pesquisa nas diferentes etapas, incluindo idade, sexo, escolaridade e condição (acompanhante versus paciente). Após a intervenção educativa, $84 \%$ dos participantes da segunda etapa versus $22,7 \%$ da primeira etapa declararam saber o que são Cuidados Paliativos $(p<0,0001)$. No que se refere à percepção sobre Cuidados Paliativos daqueles que afirmaram conhecer o tema, não houve diferença significativa entre as etapas em 13 das 14 perguntas realizadas. Conclusões: Este trabalho, além de trazer informações importantes sobre a falta de conhecimento dos pacientes e acompanhantes sobre Cuidados Paliativos, propõe um método educativo simples através de uma cartilha em formato de gibi. Os resultados apresentados mostraram-se promissores nesta população, devendo ser aprimorado e validado em outros serviços com características semelhantes.
\end{abstract}

Palavras-chave: Cuidados Paliativos; Oncologia; Acompanhantes de Pacientes.

\begin{abstract}
Objectives: To assess the understanding of cancer patients and their caregivers about palliative care before and after educational intervention, identifying the effectiveness of informative booklet application elaborated specifically for this project and seeking its validation as an educational tool. Methods: The study was divided into two stages: diagnosis and intervention. In the first, a questionnaire was applied to cancer patients and their caregivers in order to assess their level of knowledge about palliative care. From the results of the questionnaire, an educational primer was developed. In the second stage, the booklets were given to the participants of the study and, after reading them, the same questionnaire used in the first stage was applied. Results: We included 194 participants in the first stage and an additional 50 in the second stage. There weren't any difference between participants features from the first and second stages, including age, gender, schooling or condition (patient versus caregiver). After educational tool application, $84 \%$ of participants in the second stage versus $22.7 \%$ in the first stage said "yes" to the question "Do you know what palliative care is?" ( $p<0,0001)$. Of those who answered "yes", there weren't any statistical difference
\end{abstract}

Conflito de interesse: Os autores declararam não haver conflitos de interesse que precisam ser informados. 
between stages to the answers for 13 of 14 questions about palliative care. Conclusion: The current study brings important information about the population level of knowledge about palliative care. The educational booklet contributes to increase this level, however this booklet needs to be improved and tested in similar populations of other institutions.

Keywords: Palliative Care, Medical Oncology, Medical Chaperones.

\section{INTRODUÇÃO}

O termo cuidados paliativos se refere a um tratamento no qual o objetivo não é a cura do paciente, mas a melhora de sua qualidade de vida. De acordo com a definição da Organização Mundial da Saúde (OMS), trata-se de "um enfoque que busca melhorar a qualidade de vida dos pacientes e suas famílias ao afrontar os problemas associados com uma enfermidade com potencial mortal, graças à prevenção e ao alívio do sofrimento por meio da identificação antecipada, da avaliação e do tratamento impecável da dor e outros problemas físicos, psicossociais e espirituais". ${ }^{1}$ A intervenção é feita por uma equipe multidisciplinar integrada com objetivo de ajudar o paciente a se adaptar às mudanças de vida decorrentes da doença e às suas incapacitações, além de auxiliá-lo a aceitar o presente quadro junto a sua família.

De acordo com as estatísticas de 2012 da Worldwide Hospice Palliative Care Aliance (WHPCA), a cada ano mais de 20 milhões de pessoas necessitam de cuidados paliativos ao fim da vida. ${ }^{2}$ Em relação ao Brasil, a Associação Latino-Americana de Cuidados Paliativos (ALCP) publicou um Atlas em $2012^{3}$ demonstrando que há uma quantidade reduzida de instituições e de profissionais especializados em cuidados paliativos, apesar de o Conselho Federal de Medicina considerá-los eticamente obrigatórios em pacientes terminais, de haver um plano nacional que os inclui no Sistema Único de Saúde e de existirem portarias que os julgam como pré-requisitos para o funcionamento dos centros de atenção em Oncologia. Diante disso, é possível questionar o conhecimento da população brasileira acerca do tema.

Através de pesquisas na literatura, constatou-se que houve uma crescente na publicação de trabalhos relacionados a cuidados paliativos, principalmente no que diz respeito ao conhecimento dos profissionais e às formas de comunicação com os familiares e pacientes. Entretanto, poucos trabalhos foram encontrados acerca da compreensão dos doentes e da população em geral sobre o assunto. Além disso, não havia nenhum estudo brasileiro, o que revela uma lacuna importante, uma vez que a informação é essencial para ampliar o acesso aos serviços e para levar o paciente a considerar essa opção de tratamento. Afinal, a falta de conhecimento, muitas vezes, é responsável pela criação de uma imagem equivocada e negativa dos cuidados paliativos. ${ }^{4}$

Considerando a lacuna na literatura brasileira a respeito do tema, este estudo visa identificar quais informações são desconhecidas pelos pacientes e acompanhantes a respeito dos cuidados paliativos. A partir destes dados, elaborar cartilha educativa e testá-la na mesma população.

\section{MÉTODOS}

O estudo foi dividido em duas etapas: diagnóstica e de intervenção. Na primeira, aplicou-se um questionário a pacientes oncológicos e seus acompanhantes a fim de avaliar seu nível de conhecimento acerca dos Cuidados Paliativos. Com base nas respostas obtidas, uma cartilha educativa no formato de gibi foi elaborada. Na segunda etapa, as cartilhas foram entregues a novos indivíduos, aplicando-se a seguir o mesmo questionário utilizado na primeira etapa.

Após a submissão e aprovação do Comitê de Ética em Pesquisa da Faculdade de Medicina do ABC, foram convidados a participar do estudo pacientes oncológicos e seus respectivos acompanhantes do Hospital Estadual Mário Covas e do Hospital de Ensino Padre Anchieta, os quais eram maiores de 18 anos, alfabetizados e capazes de preencher os questionários sem auxílio. Apenas os cuidadores que tinham vínculo afetivo com o paciente e estavam envolvidos com o seu tratamento eram considerados acompanhantes, podendo ser um familiar ou não.

Inicialmente foi realizada uma aplicação experimental do questionário para uma amostra de 20 pacientes e seus acompanhantes, respeitando os critérios de inclusão, com a finalidade de avaliar o grau de compreensão e a aplicabilidade do teste, sendo realizadas as devidas correções. Posteriormente à aplicação experimental, foi realizada a etapa de diagnóstico, com visitas aos ambulatórios duas vezes por semana, ao longo de 10 semanas 
consecutivas, durante os meses de fevereiro a abril de 2016, até atingir 200 participantes. Eram convidados os primeiros pacientes consecutivos dos serviços de saúde selecionados e seus acompanhantes, caso houvesse. Vale ressaltar que, aqueles que respondiam "não" para a questão "Você sabe o que são cuidados paliativos?" interrompiam as respostas do questionário e os entregava ao pesquisador, entretanto, os dados já fornecidos não eram descartados.

Durante a etapa de intervenção, que ocorreu entre novembro e dezembro de 2017 no Hospital Estadual Mario Covas, inicialmente foi entregue ao participante do estudo a ferramenta educativa, cujo conteúdo foi transmitido por meio de uma história em quadrinhos com linguagem simples e objetiva. Após a sua leitura, foi realizada a aplicação do questionário no paciente ou seu acompanhante até atingir 50 participantes, de modo que fossem respeitados os critérios de inclusão utilizados na etapa anterior. Ao final da aplicação dos questionários, foi realizada uma análise quantitativa e qualitativa dos resultados, além da comparação entre os dados obtidos nas etapas de diagnóstico e intervenção.

Todos os participantes concordantes assinaram um Termo de Consentimento Livre Esclarecido, após uma breve explicação sobre o estudo e resolução de possíveis dúvidas, deixando claro o seu caráter voluntário.

\section{ANÁLISE ESTATÍSTICA}

Com o intuito de avaliar e descrever o conhecimento dos pacientes oncológicos e seus acompanhantes a respeito de cuidados paliativos, optou-se por utilizar as frequências relativa e absoluta. Além disso, comparou-se os dados obtidos na etapa de intervenção com aqueles encontrados na etapa anterior em busca de variações nos percentuais. A associação entre variáveis categóricas foi verificada através do teste do qui quadrado ou Teste Exato de Fish quando os grupos a serem comparados apresentavam porcentagem menor do que 5. Para a comparação de médias utilizou-se o teste T. O programa estatístico utilizado foi o Stata, versão 12.1.

\section{RESULTADOS}

No período de fevereiro a abril de 2016 foram incluídos 200 pacientes, sendo 6 deles excluídos por problemas no preenchimento dos questionários ou termos de consentimento com falta de assinatura. A maioria dos entrevistados era do sexo feminino, sendo $59,79 \%$ na primeira etapa e $64 \%$ do total na segunda etapa. Além disso, a maior parte da população estudada apresentava um nível de escolaridade correspondente ao ensino fundamental ou médio.

Não houve diferenças significativas em nenhuma das características entre os participantes da primeira e da segunda etapas. A Tabela 1 resume as características sócio-demográficas da população estudada.

TABELA 1- Características dos sujeitos de pesquisas incluídos na primeira e segunda etapas do estudo.

\begin{tabular}{|c|c|c|c|}
\hline & $\begin{array}{l}\text { ETAPA } 1 \\
(n=194)\end{array}$ & $\begin{array}{l}\text { ETAPA } 2 \\
(n=50)\end{array}$ & Significância estatística \\
\hline Idade (Cl 95\%) & $54,68(52,5-56,9)$ & $50,7(46,5-54,9)$ & $p=0,105^{*}$ \\
\hline \multicolumn{4}{|l|}{ Sexo } \\
\hline Feminino & $116(59,79 \%)$ & 32 (64\%) & \\
\hline Masculino & $78(40,21 \%)$ & $18(36 \%)$ & $p=0,587^{* *}$ \\
\hline \multicolumn{4}{|l|}{ Escolaridade } \\
\hline Fundamental ou Médio & $153(78,87 \%)$ & $39(78 \%)$ & \\
\hline Superior & $41(21,13 \%)$ & $11(22 \%)$ & $p=0,894^{* *}$ \\
\hline \multicolumn{4}{|l|}{ Religião } \\
\hline Católico & $102(53,12 \%)$ & $19(45,24 \%)$ & \\
\hline Evangélico & $62(32,30 \%)$ & $12(28,57 \%)$ & \\
\hline Outras & $13(6,77 \%)$ & $7(16,67 \%)$ & \\
\hline Sem religião & $15(7,81 \%)$ & $4(9,52 \%)$ & $p=0,208^{* * *}$ \\
\hline
\end{tabular}


TABELA 1- Continuacão...

\begin{tabular}{|c|c|c|c|}
\hline & $\begin{array}{l}\text { ETAPA } 1 \\
(n=194)\end{array}$ & $\begin{array}{c}\text { ETAPA } 2 \\
(n=50)\end{array}$ & Significância estatística \\
\hline \multicolumn{4}{|l|}{ Estado Civil } \\
\hline Solteiro & $118(61,14 \%)$ & $34(68 \%)$ & \\
\hline Casado & $32(16,58 \%)$ & $11(22 \%)$ & \\
\hline Viúvo & $19(9,84 \%)$ & $3(6 \%)$ & \\
\hline Divorciado & $24(12,44 \%)$ & $2(4 \%)$ & $p=0,246 * * *$ \\
\hline \multicolumn{4}{|l|}{ Entrevistado } \\
\hline Paciente & $107(55,15 \%)$ & 25 (50\%) & \\
\hline Acompanhante & 87 (44,85\%) & $25(50 \%)$ & $\mathrm{p}=0,514^{* *}$ \\
\hline
\end{tabular}

* Teste $\mathrm{T}$; ** Teste Chi2; *** Teste Exato de Fish

Quanto ao conhecimento dos entrevistados sobre os cuidados paliativos é preciso destacar alguns resultados encontrados. Apenas $22,68 \%$ dos pacientes afirmaram saber ou pelo menos ter uma leve ideia sobre o que viria a ser os cuidados paliativos. Dentre os que sabiam, $20,45 \%$ acreditavam que era um tratamento com intuito curativo, $34 \%$ que aumentaria o tempo de vida, $9,09 \%$ que atrasavam o tratamento e $75 \%$ achava que não tiravam os sintomas. Todos os dados a respeito do conhecimento dos entrevistados sobre cuidados paliativos na primeira etapa estão explicitados na Tabela 2.

TABELA 2 - Conhecimento dos entrevistados da primeira etapa a respeito de Cuidados Paliativos

\begin{tabular}{|c|c|c|c|c|c|c|}
\hline \multirow{2}{*}{ Sobre os cuidados paliativos } & \multicolumn{2}{|c|}{ Sim } & \multicolumn{2}{|c|}{ Não } & \multicolumn{2}{|c|}{ Não Sabe responder } \\
\hline & $\mathbf{N}$ & $\%$ & $\mathbf{N}$ & $\%$ & $\mathbf{N}$ & "\% \\
\hline Sabe o que são? & 44 & 22,68 & 150 & 77,32 & 0 & 0 \\
\hline Conhece alguém que recebeu? & 29 & 65.91 & 15 & 34.09 & 0 & 0 \\
\hline São somente para pacientes com câncer? & 3 & 6.82 & 41 & 93.18 & 0 & 0 \\
\hline $\begin{array}{l}\text { São apenas para pacientes em seus últimos dias de } \\
\text { vida? }\end{array}$ & 2 & 4,55 & 42 & 95,45 & 0 & 0 \\
\hline Servem para alivia a dor? & 42 & 95,45 & 2 & 4,55 & 0 & 0 \\
\hline Servem para curar a doença? & 9 & 20,45 & 35 & 79,55 & 0 & 0 \\
\hline Servem para melhora a qualidade de vida? & 43 & 97,73 & 1 & 2,27 & 0 & 0 \\
\hline $\begin{array}{l}\text { São tratamentos realizados junto com outros } \\
\text { (radioterapia, quimioterapia, cirrugia, etc)? }\end{array}$ & 41 & 93,18 & 2 & 4,55 & 1 & 2,27 \\
\hline Usados somente em pacientes idosos? & 1 & 2,27 & 43 & 97,73 & 0 & 0 \\
\hline $\begin{array}{c}\text { Servem apenas para o paciente, e não para seus } \\
\text { familiares? }\end{array}$ & 9 & 20,45 & 35 & 79,55 & 0 & 0 \\
\hline Podem ajudar a família? & 43 & 97,73 & 1 & 2,27 & 0 & 0 \\
\hline Podem atrasar o tratamento? & 4 & 9,09 & 40 & 90,91 & 0 & 0 \\
\hline Tiram os sintomas? & 11 & 25 & 33 & 75 & 0 & 0 \\
\hline Uso exclusivo de remédios? & 11 & 25 & 33 & 75 & 0 & 0 \\
\hline Melhoram a forma da morte? & 35 & 79,55 & 9 & 20,45 & 0 & 0 \\
\hline Trazem conforto espiritual? & 38 & 86,36 & 6 & 13,64 & 0 & 0 \\
\hline
\end{tabular}


TABELA 3 - Graus de conhecimento declarado acerca de cuidados paliativos antes e após intervenção educativa através da cartilha.

\begin{tabular}{|c|c|c|c|}
\hline & $\begin{array}{l}\text { ETAPA } 1 \\
(n=194)\end{array}$ & $\begin{array}{l}\text { ETAPA } 2 \\
(n=50)\end{array}$ & Significância estatística \\
\hline \multicolumn{4}{|c|}{ Sabe o que são cuidados paliativos? } \\
\hline Sim ou leve ideia & $44(22,68 \%)$ & $42(84 \%)$ & \\
\hline Não & $150(77,32 \%)$ & $8(16 \%)$ & $p<0,0001 *$ \\
\hline * Teste Chi2 & & & \\
\hline
\end{tabular}

Com base nos resultados apresentados na Tabela 2, elaborou-se um cartilha educativa em formato de gibi. A fim de facilitar a leitura para uma população com escolaridade correspondente a ensino fundamental e médio na sua maioria, utilizou-se frases curtas e letras grande, bem como vocabulário simples e coloquial. A cartilha final pode ser vista no ANEXO 1.

No período de novembro a dezembro de 2017 foram incluídos 50 pacientes na segunda etapa do estudo. Após a leitura das cartilhas, observamos um aumento significativo entre aqueles que declaravam saber o seriam os cuidados paliativos (TABELA 3).

Ao analisarmos cada uma das respostas dadas por aqueles que declaravam conhecer o que viriam a ser os cuidados paliativos, observamos que não houve diferença significativa entre as etapas para 13 das 14 perguntas realizadas (TABELA 4).

TABELA 4 - Percepção sobre cuidados paliativos daqueles que afirmaram conhecer o tema.

\begin{tabular}{|c|c|c|c|}
\hline & $\begin{array}{l}\text { ETAPA } 1 \\
(n=44)\end{array}$ & $\begin{array}{l}\text { ETAPA } 2 \\
(n=42)\end{array}$ & $\begin{array}{l}\text { Significância } \\
\text { estatística }\end{array}$ \\
\hline \multicolumn{4}{|c|}{ São somente para pacientes com câncer? } \\
\hline Sim & $3(6,82 \%)$ & $0(0 \%)$ & \\
\hline Não & $41(93,18 \%)$ & $42(100 \%)$ & $p=0,242 * *$ \\
\hline \multicolumn{4}{|c|}{ São apenas para pacientes em seus últimos dias de vida? } \\
\hline Sim & $2(4,55 \%)$ & $0(0 \%)$ & \\
\hline Não & $42(95,45 \%)$ & $42(100 \%)$ & $p=0,494 * *$ \\
\hline \multicolumn{4}{|c|}{ Servem para aliviar a dor? } \\
\hline Sim & $42(95,45 \%)$ & $36(85,71 \%)$ & \\
\hline Não & $2(4,55 \%)$ & $6(14,29 \%)$ & $p=0,152$ \\
\hline \multicolumn{4}{|c|}{ Servem para curar a doença? } \\
\hline Sim & $9(20,45 \%)$ & $11(26,83 \%)$ & \\
\hline Não & $35(79,55 \%)$ & $30(73,17 \%)$ & $p=0,489 *$ \\
\hline \multicolumn{4}{|c|}{ Servem para melhorar a qualidade de vida? } \\
\hline Sim & $43(97,73 \%)$ & $42(100 \%)$ & \\
\hline Não & $1(2,27 \%)$ & $(0 \%)$ & $p=1,000 * *$ \\
\hline \multicolumn{4}{|c|}{ Servem para melhorar a saúde emocional/mental? } \\
\hline Sim & $44(100 \%)$ & $39(92,86 \%)$ & \\
\hline Não & $0(0 \%)$ & $3(7,14 \%)$ & $p=1,112^{* *}$ \\
\hline \multicolumn{4}{|c|}{$\begin{array}{l}\text { São tratamentos realizado juntamente com outros (radioterapia, } \\
\text { quimioterapia, cirurgia, etc)? }\end{array}$} \\
\hline Sim & $42(95,45 \%)$ & $35(83,33 \%)$ & \\
\hline Não & $2(4,55 \%)$ & $7(16,67 \%)$ & $p=0,066^{*}$ \\
\hline \multicolumn{4}{|c|}{ Usados somente em pacientes idosos? } \\
\hline Sim & $1(2,27 \%)$ & $0(0 \%)$ & \\
\hline
\end{tabular}


TABELA 4 - Continuação...

\begin{tabular}{|c|c|c|c|}
\hline & $\begin{array}{l}\text { ETAPA } 1 \\
(n=44)\end{array}$ & $\begin{array}{l}\text { ETAPA } 2 \\
(n=42)\end{array}$ & $\begin{array}{c}\text { Significância } \\
\text { estatística }\end{array}$ \\
\hline Não & $43(97,73 \%)$ & $42(100 \%)$ & $\mathrm{p}=1,000 * *$ \\
\hline \multicolumn{4}{|c|}{ Servem apenas para o paciente, e não para seus familiares? } \\
\hline Sim & $9(20,45 \%)$ & $8(19,05 \%)$ & \\
\hline Não & $35(79,55 \%)$ & $34(80,95 \%)$ & $p=0,870^{*}$ \\
\hline \multicolumn{4}{|l|}{ Podem ajudar a família? } \\
\hline Sim & $43(97,73 \%)$ & $41(97,62 \%)$ & \\
\hline Não & $1(2,27 \%)$ & $1(2,38 \%)$ & $p=1,000 * *$ \\
\hline \multicolumn{4}{|c|}{ Podem atrasar o tratamento? } \\
\hline Sim & $4(9,10 \%)$ & $2(4,76 \%)$ & \\
\hline Não & $40(90,90 \%)$ & $40(95,24 \%)$ & $p=0,677^{* *}$ \\
\hline \multicolumn{4}{|l|}{ Uso exclusivo de remédios? } \\
\hline Sim & $11(25 \%)$ & $13(31,71 \%)$ & \\
\hline Não & $33(75 \%)$ & $28(68,29 \%)$ & $p=0,492^{*}$ \\
\hline \multicolumn{4}{|c|}{ Melhoram a forma da morte? } \\
\hline Sim & $35(79,55 \%)$ & $23(54,76 \%)$ & \\
\hline Não & $9(20,45 \%)$ & $19(45,24 \%)$ & $p=0,014^{*}$ \\
\hline \multicolumn{4}{|l|}{ Trazem conforto espiritual? } \\
\hline Sim & $38(86,36 \%)$ & $38(90,48 \%)$ & \\
\hline Não & $6(13,64 \%)$ & $4(9,52 \%)$ & $p=0,739 * *$ \\
\hline \multicolumn{4}{|l|}{ * Teste Chi2 } \\
\hline ** Teste Exato de Fish & & & \\
\hline
\end{tabular}

\section{DISCUSSÃO}

Os dados do trabalho revelaram, de modo geral, que na etapa de diagnóstico a maioria dos entrevistados, tanto pacientes como acompanhantes, não sabia o que seriam os cuidados paliativos. Para a pergunta "Você sabe o que são cuidados paliativos?", dos 194 entrevistados, com um intervalo de idade que variava de 20 a 93 anos, 147 (76.17\%) responderam "não" enquanto que 44 (22.68\%) responderam "sim" ou "tenho uma leve ideia", sendo que apenas 1 indivíduo respondeu a todas as questões de forma correta $(<0,51 \%)$, revelando que existe uma grande deficiência no conhecimento ou entendimento sobre o tema em questão.

Já na etapa de intervenção, após a leitura da cartilha, a maioria dos entrevistados sabia o que seriam cuidados paliativos (84\%), representando um incremento significativo $(p<0,0001)$.

Um estudo italiano ${ }^{5}$ que avaliou a percepção dos cuidados paliativos realizado aleatoriamente com indivíduos entre 18 e 74 anos mostrou que nesta população o conhecimento do tema é inadequado, sendo que menos de $60 \%$ dos participantes já tinham ouvido falar sobre cuidados paliativos, enquanto que apenas $7 \%$ destes acreditavam ter uma ideia precisa sobre o que realmente são os cuidados. Esse resultado se assemelha ao encontrado na primeira etapa do projeto e reforça a importância de uma intervenção educativa direcionada às lacunas de conhecimento da população sobre esse tratamento cada vez mais necessário e relevante.

Quando analisamos apenas os indivíduos que declaravam saber o que seriam os cuidados paliativos, tanto na primeira como na segunda etapas, percebemos que não houve diferenças significativas entre a natureza das respostas para a quase totalidade das perguntas (13 de 14 questões). Este achado indica para um real ganho de conhecimento entre aqueles que, após a leitura da cartilha, passaram a se declarar conhecedores do tema. Apesar disso, nota-se que em ambas as etapas ainda há um número expressivo de indivíduos que apresenta erros 
conceituais sobre o tema, em questões aparentemente simples como "Servem para curar a doença?" ou "Uso exclusivo de remédios?".

Este fato mostra que, apesar de representar um avanço como instrumento educacional, a cartilha ainda carece de ajustes a fim de sanar tais erros de entendimento. Além disso, vale ressaltar que os resultados obtidos têm a limitação relacionada ao tamanho reduzido da amostra, bem como o fato do estudo ter sido realizado em um único serviço que atende apenas pacientes oncológicos.

Este trabalho, além de trazer informações importantes sobre a falta de conhecimento dos pacientes e acompanhantes sobre Cuidados Paliativos, propõe um método educativo simples através de uma cartilha em formato de gibi. Os resultados apresentados mostraram-se promissores nesta população, devendo ser aprimorado e validado em outros serviços com características semelhantes.

\section{REFERÊNCIAS BIBLIOGRÁFICAS}

1. World Health Organization. Palliative care: the solid facts. Geneva: WHO; 2004.

2. Connor S, Bermedo MC. WHO global atlas on palliative care at end of life. 1st ed. Londres: Worldwide Palliative Care Alliance; 2014. 102 p.

3. Pastrana T. et al. Atlas de cuidados paliativos na America Latina. Houston: IHPC press; 2012. 55 p.

4. Wallace J. Public awareness of palliative care: report of the findings of the first national survey in Scotland into public knowledge and understanding of palliative care. Edinburgh: Schottish Partnership for Palliative Care; 2013. 24 p.

5. Benini F, Fabris M, Pace DS, et al. Awareness, understanding and attitudes of Italians regarding palliative care. Ann Ist Super Sanità. 2011;47(3):253-9. PMid:21952149. 
Anexo 1 - Cartilha educativa em formato de gibi utilizada na etapa de intervenção do estudo
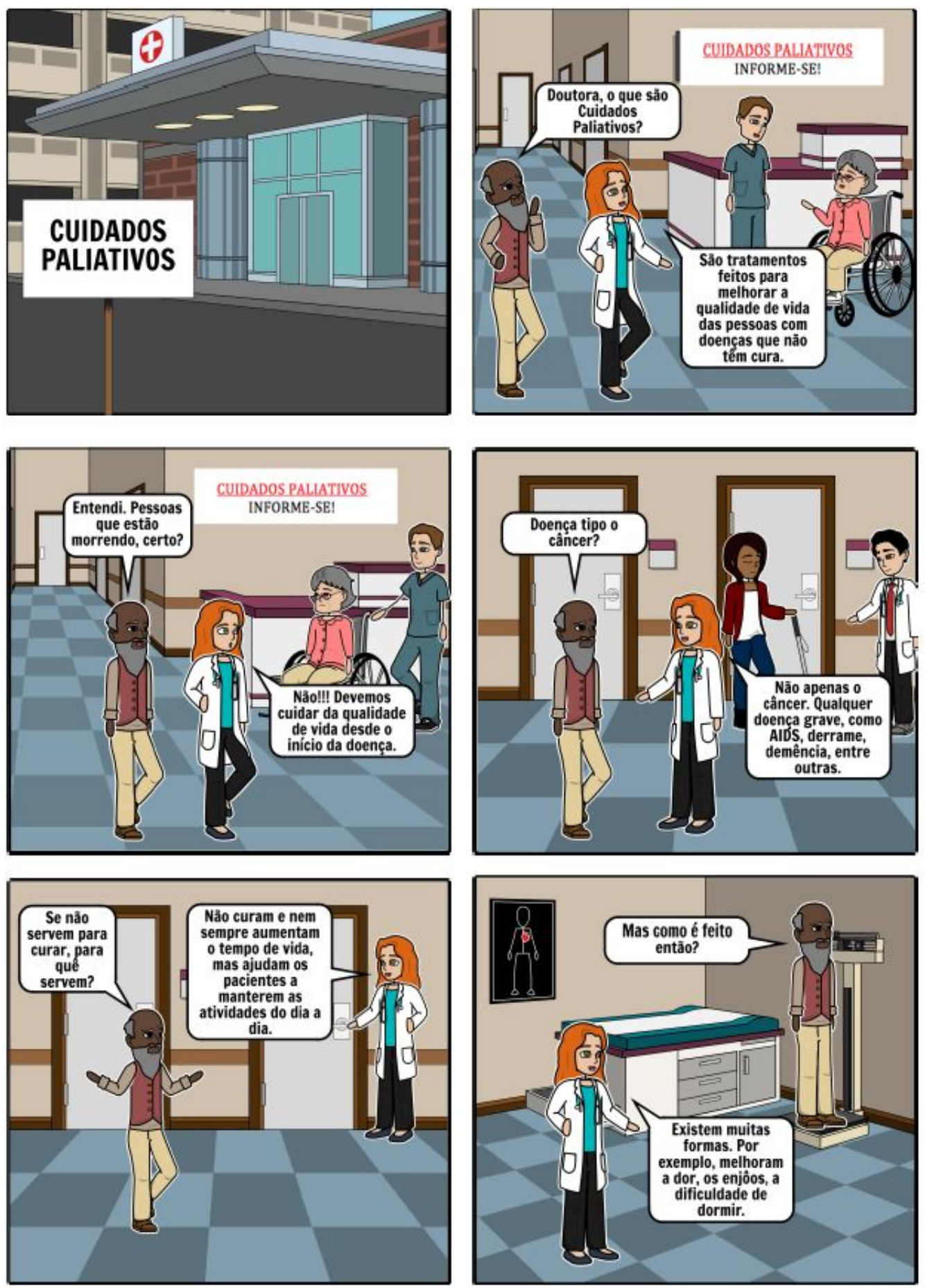

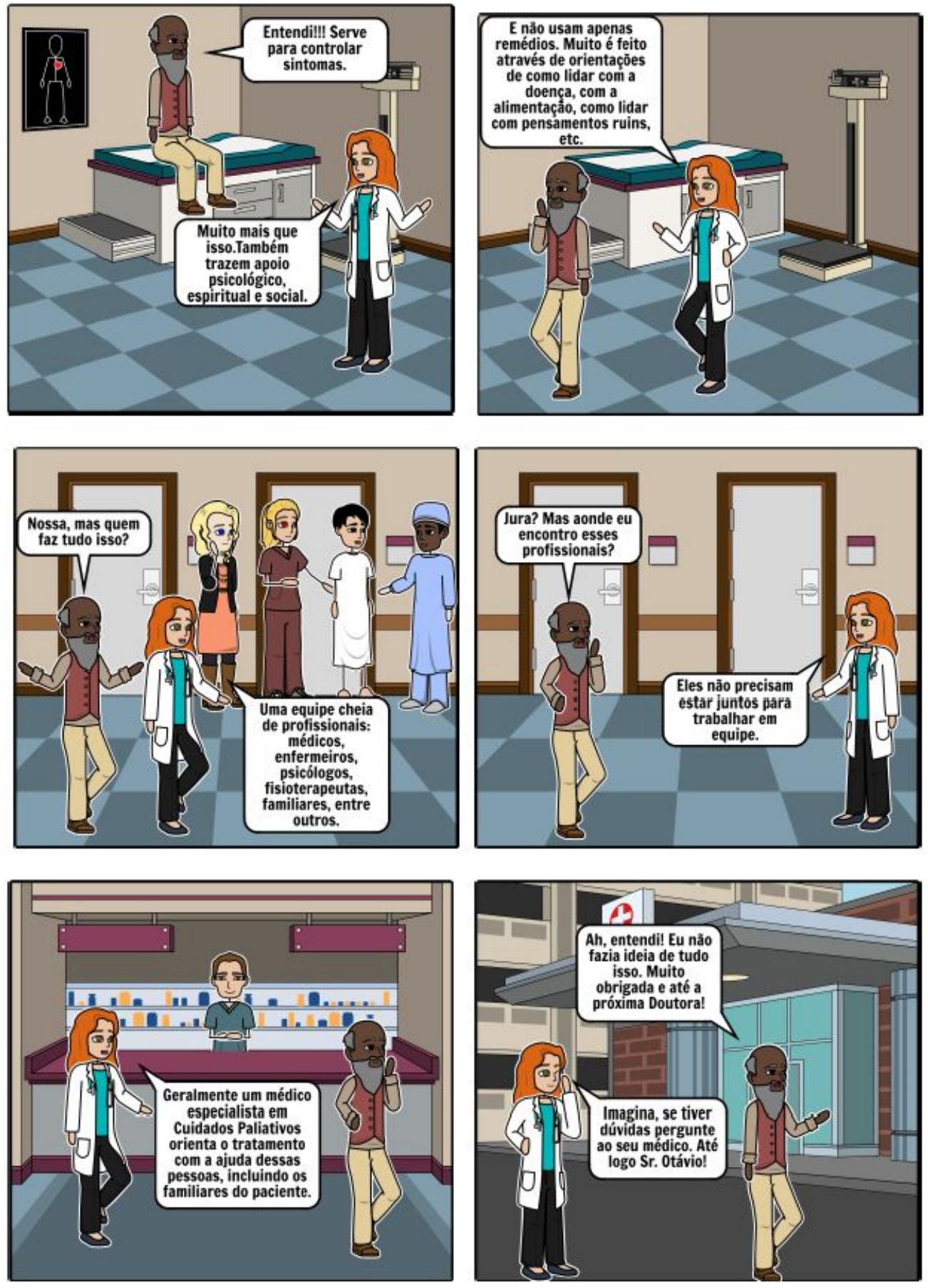